\title{
Determinação das fontes alimentares de Anopheles aquasalis (Diptera: Culicidae) no Estado do Rio de Janeiro, Brasil, pelo teste de precipitina*
}

\section{Blood-meal sources of Anopheles aquasalis (Diptera: Culicidae) in a South-eastern State of Brazil}

\author{
Carmen Flores-Mendoza, Rodolfo A. Cunha, Dayse S. Rocha e Ricardo Lourenço-de- \\ Oliveira \\ Laboratório de Transmissores de Hematozoários do Instituto Oswaldo Cruz. Rio de Janeiro, RJ - \\ Brasil (C. F. M., R. L.O.), Laboratório Nacional e Internacional de Taxonomia de Triatomíneos do \\ Instituto Oswaldo Cruz. Rio de Janeiro, RJ - Brasil (R. A. C., D. S. R.)
}

\begin{abstract}
Resumo
Anopheles aquasalis é um mosquito ora encarado como antropofílico, ora como zoofílico ou eclético. Realizou-se estudo em Guapimirim, Estado do Rio de Janeiro, de maio a novembro de 1992, com o intuito de se verificar a fonte alimentar preferida desse anofelino através de teste imunológico de precipitina. De 1.366 fêmeas capturadas em abrigos naturais, 725 estavam ingurgitadas. O conteúdo digestivo de apenas 473 delas reagiu no teste de precipitina, sendo que em $75,3 \%$ dos casos foi identificada apenas uma fonte alimentar. Mais da metade dessas fêmeas havia se alimentado em boi $(52,2 \%)$, enquanto poucas tinham sugado homem $(1,1 \%)$. Por outro lado, $24,7 \%$ dos espécimes haviam se alimentado em mais de uma fonte sangüínea, principalmente boi e cavalo. Conclui-se que An. aquasalis é zoófilo nessa região do País, utilizando grande variedade de hospedeiros, porém preferindo se alimentar em animais de grande porte, especialmente o boi e cavalo.
\end{abstract}

Anopheles. Preferências alimentares. Ecologia de vetores.

\begin{abstract}
Anopheles aquasalis has shown local variations in blood-host preference in Brazil: it seems to be anthropophilic in the northeast and zoophilic or opportunistic in the Amazon and other regions. A study was carried out in Guapimirim county, State of Rio de Janeiro, from May to November 1992, for the purpose of identifying the blood meal source of this anopheline by capillary tube precipitin test. A total of 1,366 females were captured at natural resting-places, 725 of which were blood-fed. The gut content of 473 blood fed females reacted to the antisera used (human, cow, horse, pig, dog and chicken). The majority of the females - 356 (75.3\%) - had blood from
\end{abstract}

* Subvencionado pelo Conselho Nacional de Desenvolvimento Científico e Tecnológico/CNPq/FIOCRUZ e pelo convênio Fundação Nacional de Saúde/ FIOCRUZ/BIRD-027/93.

Parte da Dissertação de Mestrado de C. Flores-Mendoza, apresentada à FIOCRUZ.

Correspondência para/Correspondence to: Carmen Flores-Mendoza - Laboratório de Transmissores de Hematozoários do Instituto Oswaldo Cruz. Av. Brasil, 4365 - 21045-900 Rio de Janeiro, RJ - Brasil. Fax: (021)290.9339 E-mail:ffrl@ DCCØØ1.CICT.FIOCRUZ.BR

Recebido em 2.6.1995. Aprovado em 9.1.1996. 
only one source. A substantial bovine preference was observed - 52.2\% had fed on cows, $29.8 \%$ on horses, $10.7 \%$ on pigs, $4.5 \%$ on chickens and $1.7 \%$ on dogs, but only few had fed on man (1.1\%) and none on rats. On the other hand, $24.7 \%$ of the females had fed on more than one host, mainly on cows and horses. It was assumed that An. aquasalis is zoophilic in southeastern Brazil, feeding on a wide variety of animals, the cow being the primary host.

Anopheles. Food preferences. Ecology, vectors.

\section{INTRODUÇÃO}

Um dos parâmetros mais utilizados na compreensão da epidemiologia e no estudo da dinâmica da transmissão do paludismo é a determinação da capacidade vetorial (CV) (Dye $\left.{ }^{17}, 1986\right)$. É indispensável, na equação que define a $\mathrm{CV}$, conhecer-se a proporção de fêmeas da espécie encontradas naturalmente ingurgitadas com sangue humano e de outros animais. De acordo com essa equação, quanto menor a proporção de fêmeas de uma dada espécie encontradas com sangue humano, menor será a sua capacidade vetorial e vice-versa (Garret e Jones ${ }^{25}$, 1964).

Anopheles (Nys.) aquasalis, importante vetor litorâneo da malária em diversas localidades do Brasil (Fig. 1) e Américas (Senior-White ${ }^{33}$, 1952; Gabaldón \& Guerrero $^{23}$, 1959; Rachou ${ }^{29}$, 1958; Grossi, 1960 (apud Cova-Garcia e col. ${ }^{12}, 1964$ ); Deane ${ }^{14}$, 1986; Berti e col. ${ }^{4}, 1993$ ), vem apresentando comportamento alimentar bastante variável. Em Belém, na Amazônia brasileira, onde o clima é equatorial, o mosquito é preferentemente zoófilo (Galvão e col. ${ }^{24}, 1942$ ), ao passo que no Nordeste, de clima semi-árido, é mais antropófilo (Deane e col. ${ }^{15}$, 1948; Rachou e col. ${ }^{30}$, 1950). Já no Sudeste do Brasil, cujo clima é semitropical, tem sido observado ora atacando o homem ora os animais domésticos ao ar livre (Coutinho ${ }^{7,8,9}$, 1942, 1943, 1946; Ramos $^{31}$, 1943; Coutinho e Ricciardi $^{11}$, 1943; Lourenço-de-Oliveira e Heyden ${ }^{27}$, 1986). Na Guiana e no Oeste panamenho, verificouse que é um mosquito muito zoófilo, não sendo encarado como de importância epidemiológica (Curry $^{13}$, 1932; Gigliolii ${ }^{26}$, 1940; Rozeboom ${ }^{32}$, 1942).

Em vista das divergências no hábito hematofágico de An. aquasalis no País, decidiu-se verificar qual é a sua principal fonte alimentar em uma localidade na Baixada Fluminense, região Sudeste, onde esse é o único anofelino abundante, tendo sido considerado vetor potencial durante pequenas epidemias de malária ali registradas nas duas últimas décadas (Flores-Mendoza $\left.{ }^{19}, 1994\right) *$.

\section{MATERIAL E MÉTODO}

As coletas foram realizadas numa propriedade rural, com atividade pecuária leiteira - fazenda "Meia Noite" - em Guaraí, (2241'S 4257'W), no Município de Guapimirim, Estado do Rio de Janeiro, duas ou três vezes por mês, no período de maio a novembro de 1992, no horário das 9:00 às 13:00 h.

Os mosquitos foram procurados e capturados fora do perímetro de $500 \mathrm{~m}$ do conjunto da casa e o pequeno estábulo da fazenda, em locais com características semelhantes às descritas para os abrigos de mosquitos por Shannon $^{34}$ (1935), Barnes ${ }^{2}$ (1945), Bates ${ }^{3}$ (1949) e Forattini $^{21}$ (1962).

Três ecótopos foram identificados como os mais utilizados como abrigos (Flores-Mendoza e Deane ${ }^{18}$, 1993; Flores-Mendoza ${ }^{19}$, 1994) por An. aquasalis na área: moitas de capim, bananal e troncos de árvores caídos. Utilizou-se tubo aspirador manual, puçá e aparelho de sucção tipo aspirador (Natal e Marucci ${ }^{28}$, 1984).

Os mosquitos eram postos em gaiolas de papelão, rotuladas segundo a procedência e a técnica de coleta e acondicionados em caixa de isopor de modo a transportá-los vivos ao laboratório. Após triagem e identificação das fêmeas, aquelas ingurgitadas eram imediatamente armazenadas em refrigerador $\left(4^{\circ} \mathrm{C}\right)$, para impedir que se continuasse a digestão do sangue ingerido.

O conteúdo intestinal era colhido em uma tira de papel-filtro Watman $n^{\circ}$ 1, numerada, posta a secar, embrulhada com papel celofane e mantida em refrigerador até a análise pela prova de precipitina. Para esse teste, o papel-filtro contendo a amostra era mergulhado em solução salina $(\mathrm{NaCl} 0,85 \%)$ e o eluato daí obtido examinado pela reação imunológica de precipitina, através da técnica dos tubos capilares, segundo Siqueira $^{35}$ (1960).

A pesquisa das fontes sangüíneas foi realizada fazendo-se o eluato do material colhido em papel-filtro reagir contra anti-soros, dos seguintes grupos de vertebrados: (a) mamíferos: homem (Homo), boi (Bos), cavalo (Equus), cão (Canis), porco (Sus) e roedor (Rattus) e (b) ave (Gallus). A preparação dos anti-soros e a avaliação de sua titulação e especificidade foram feitas de acordo com Siqueira ${ }^{35}$ (1960). A titulação desses anti-soros foi realizada fazendo-os reagir com diluições crescentes 


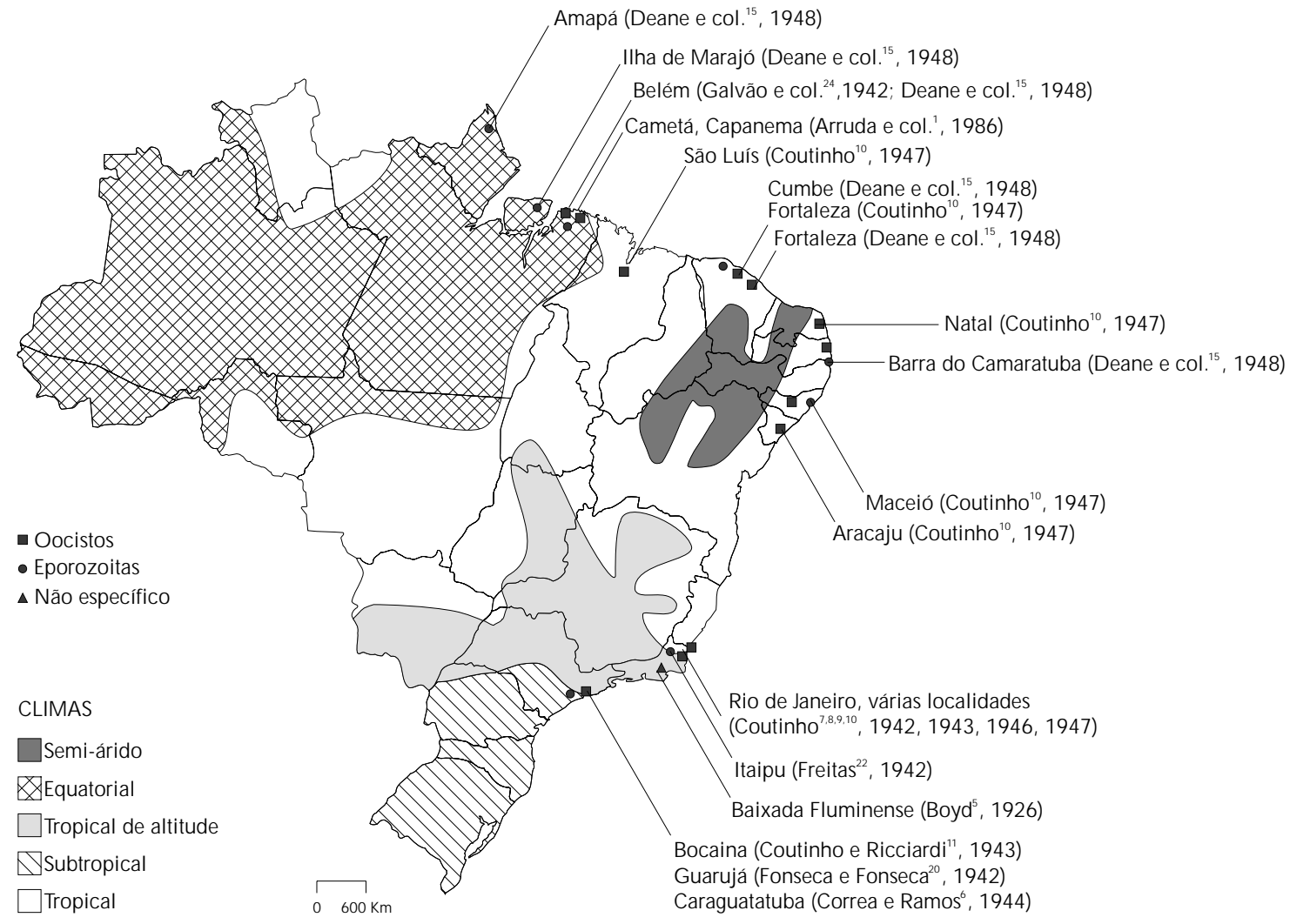

Figura 1 - Dados sobre o encontro de infecção natural, por Plasmodium, em Anopheles aquasalis, no Brasil.

dos soros homólogos de 1:5.000 a 1:12.000. Após absorção, pela adição de uma mistura de antígenos heterólogos em 100 partes de cada anti-soro, sua titulação final foi a seguinte: homem $(1: 7.000)$, ave $(1: 10.000)$, cão $(1: 10.000)$, cavalo $(1: 10.000)$, boi $(1: 9.000)$, porco $(1: 10.000)$ e roedor $(1: 10.000)$. A especificidade de cada anti-soro foi avaliada com ensaios usando-se An. aquasalis criados em laboratório, alimentados em uma ou mais fontes sangüíneas conhecidas.

\section{RESULTADOS}

Foram capturadas 1.366 fêmeas de An. aquasalis em abrigos naturais, das quais $1.211(88,6 \%)$ foram coletadas com tubo aspirador de Castro, 143 $(10,5 \%)$ com aparelho de sucção tipo aspirador e $12(0,9 \%)$ com puçá. Desse total, 725 fêmeas $(53,1 \%)$ estavam ingurgitadas. O maior número de fêmeas ingurgitadas - $618(85,2 \%)$ - foi pego em moitas de capim (Tabela 1). O conteúdo intestinal de somente $473(65,2 \%)$ reagiu ao teste de precipitina, enquanto o de 252 (34,8\%) não reagiu com os anti-soros utilizados.
Tabela 1 - Número de fêmeas não ingurgitadas e ingurgitadas, de An. aquasalis, coletadas por tipo de abrigo natural, em Guaraí, RJ, de maio a novembro de 1992.

\begin{tabular}{|c|c|c|c|c|}
\hline \multirow[t]{2}{*}{ Abrigo } & \multicolumn{2}{|c|}{$\begin{array}{c}\mathrm{N} \text { ão } \\
\text { ingurgitadas }\end{array}$} & \multicolumn{2}{|c|}{ ingurgitadas } \\
\hline & $\mathrm{N} 0$ & $\%$ & $\mathrm{~N} 0$ & $\overline{\%}$ \\
\hline Moitas de capim & 77 & 12,0 & 618 & 85,2 \\
\hline Bananal & 379 & 59,1 & 87 & 12,0 \\
\hline Tronco de árvores & 185 & 28,9 & 20 & 2,8 \\
\hline Total & 641 & 100,0 & 725 & 100,0 \\
\hline
\end{tabular}

Dentre os espécimes reagentes, 356 (75,3\%) haviam se alimentado em apenas uma fonte sangüínea (Fig. 2): mais da metade deles havia se alimentado com sangue bovino - $186(52,2 \%)-\mathrm{e}$ apenas $4(1,1 \%)$ com sangue humano. Nenhuma das amostras reagiu com o anti-soro de roedor. $\mathrm{O}$ conteúdo intestinal de 117 espécimes $(24,7 \%)$ reagiu com mais de uma fonte sangüínea (Tabela 2), sendo mais freqüente a combinação boi + cavalo $(83,7 \%)$, seguida de longe por homem + boi $(5,1 \%)$, dentre outras. 
Tabela 2 - Identificação do sangue ingerido por fêmeas de An. aquasalis, que haviam se alimentado em mais de uma fonte sangüínea, coletadas em abrigos naturais, em Guaraí, RJ, de maio a novembro de 1992.

\begin{tabular}{lrr}
\hline Repastos & N 0 & $\%$ \\
\hline Homem + boi & 6 & 5,1 \\
Homem + cavalo & 2 & 1,7 \\
Boi + cavalo & 98 & 83,7 \\
Boi + porco & 2 & 1,7 \\
Boi + cão & 2 & 1,7 \\
Cavalo + cão & 3 & 2,5 \\
Cavalo + porco & 1 & 0,9 \\
Homem + boi + cavalo & 1 & 0,9 \\
Cavalo + porco + ave & 1 & 0,9 \\
Boi + cavalo + cão + porco & 1 & 0,9 \\
\hline Total & 117 & 100,0 \\
\hline
\end{tabular}

\section{DISCUSSÃO}

An. aquasalis é considerado um anofelino dotado de variável comportamento alimentar quanto à fonte sangüínea, tendo algumas vezes sido encarado como eclético ou oportunista, variando no tempo e no espaço o seu principal hospedeiro, como verificou Senior-White ${ }^{33}$ (1952), em Trinidade.

Vimos que, na área aqui estudada, An. aquasalis é capaz de sugar, espontaneamente, qualquer dos animais cujos anti-soros foram usados no teste, isto é, homem, boi, cavalo, porco, cão e aves, exceto roedor. Um total de 292 fêmeas $(82,0 \%)$ desse anofelino haviam se alimentado em boi ou cavalo (Fig. 2), demonstrando a sua preferência por animais de grande porte, já observada por outros autores (Boyd ${ }^{5}, 1926$; Deane e col. $\left.{ }^{15}, 1948\right)$.

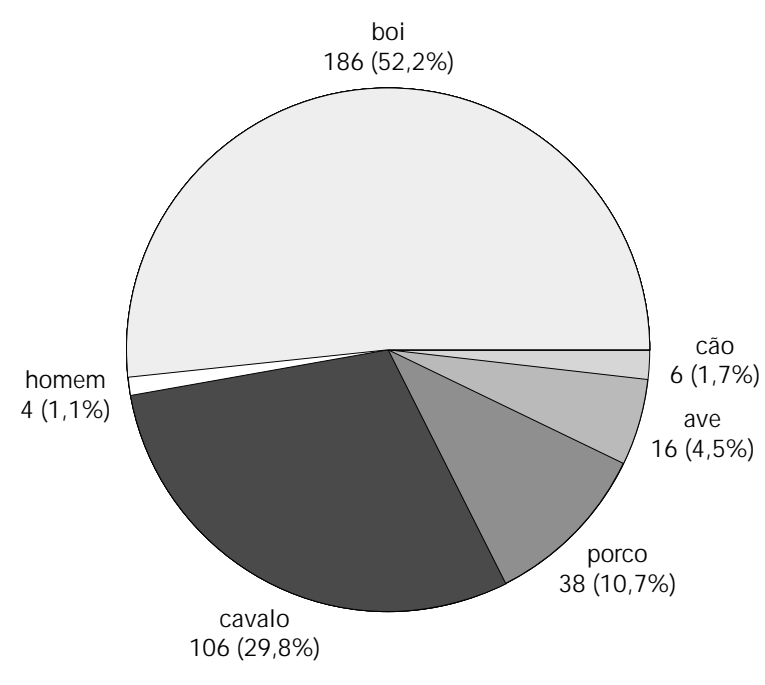

Figura 2 - Identificação do sangue ingerido por fêmeas de An. aquasalis que haviam se alimentado em apenas uma fonte sangüínea, coletadas em abrigos naturais, em Guaraí, Rio de Janeiro, de maio a novembro de 1992, através da prova de precipitina.
Deane e col. ${ }^{16}(1949)$, numa experiência feita com 1.215 An. aquasalis, procedentes de criação e soltos em uma gaiola onde pernoitaram diferentes iscas, obtiveram os seguintes resultados através de provas de precipitina: $684(56,3 \%)$ foram positivos para antisoros de boi, $284(23,4 \%)$ para cavalo, $132(10,9 \%)$ para homem, $69(5,7 \%)$ para cão, $36(3,6 \%)$ para porco, $22(1,8 \%)$ para carneiro, $21(1,7 \%)$ para gato e três $(0,4 \%)$ para galinha. De acordo com tais resultados, Deane e col. ${ }^{16}$ (1949) demonstraram que aquasalis é capaz de sugar qualquer dos animais a ele expostos, mostrando um grau de antropofilia muito pequeno em comparação a sua preferência pelo sangue de boi e de cavalo.

Lourenço-de-Oliveira e Heyden ${ }^{27}$ (1986), trabalhando no Rio de Janeiro, verificaram a nítida preferência alimentar de An. aquasalis pelo sangue de boi, ao realizarem capturas comparativas e simultâneas em cavalo, carneiro, galo e sapo. Só coletaram o anofelino em boi $(93,4 \%)$ e em cavalo $(6,6 \%)$. Aliás, Boyd $^{5}$ (1926), comparando os hábitos de An. aquasalis com os de An. darlingi na Baixada Fluminense, considerou a primeira espécie mais zoófila que a segunda.

Avaliando a preferência alimentar de An. aquasalis, em Belém, através de provas de precipitina feitas com de 596 exemplares capturados no domicílio, Deane e col $^{16}$. (1949) verificaram que 428 $(71,8 \%)$ foram positivos para anti-soro de homem, $58(9,7 \%)$ para cão, $23(3,9 \%)$ para cavalo, sete $(1,2 \%)$ para galinha, três $(0,5 \%)$ para boi e um $(0,2 \%)$ para porco. Esses autores mostraram, com suas duas experiências, que a origem do material analisado em provas de precipitina é importante, podendo resultar em falsas interpretações, pois de acordo apenas com o exame dos espécimes, coletados no domicílio, a espécie poderia ser encarada como de elevado grau de antropofilia.

No presente estudo, para evitar esse tipo de influência nos resultados, só foi analisado o conteúdo digestivo de An. aquasalis capturados fora do perímetro de $500 \mathrm{~m}$ da casa e dos abrigos dos animais domésticos (curral, pocilga, galinheiro).

Estimamou-se o número oscilante de fontes alimentares sangüíneas disponíveis na área, no período de realização do presente estudo, em: 5 a 10 humanos, 20 a 30 bovinos, 1 a 3 eqüinos, 2 a 4 cães, 1 gato, 5 a 8 galinhas e 2 a 4 porcos. Não foi quantificada a freqüência de animais silvestres, alguns dos quais comumente avistados na área, como aves, nem o número de roedores sinantrópicos.

Não se descarta a possível influência exercida nos resultados pela maior proporção de bovinos dentre os 
outros animais locais. Porém, deve-se considerar a preferência que An. aquasalis mostra pelo sangue de bovinos, mesmo quando na presença de outros animais em mesmo número, como viram Deane e col. ${ }^{16}$ (1949) e Lourenço-de-Oliveira e Heyden ${ }^{27}$ (1986).

Concluiu-se que An. aquasalis tem hábitos zoófilos, com preferência pelo sangue de boi e ca-

\section{REFERÊNCIAS BIBLIOGRÁFICAS}

1. ARRUDA, M.; CARVALHO, M.; NUSSENZWEIG, R.S.; MARACIC, M.; FERREIRA, W.; COCHRANE, A. Potential vectors of malaria and their different susceptibility to Plasmodium falciparum and Plasmodium vivax in Northern Brazil identified by immunoassay. Am. J. Trop. Med. Hyg., 35: $873-81,1986$.

2. BARNES, C. R. Anopheles walkeri in diurnal shelters in Massachusetts. J. Econ. Entomol., 38: 145, 1945.

3. BATES, M. The natural history of mosquitoes. New York, Macmillan Company, 1949.

4. BERTI, J.; ZIMMERMAN, R. H.; AMARISTA, J. Spatial and temporal distribution of anopheline larvae in two malarious areas in Sucre State, Venezuela. Mem. Inst. Oswaldo Cruz, 88: 353-62, 1993.

5. BOYD, M. F. Studies of the epidemiology of malaria in the coastal lowlands of Brazil, made before and after execution of control measures. Am. J. Hyg. Monogr. Ser, 5, 1926.

6. CORREA, R. R. \& RAMOS, A. S. Os anofelinos da Ilha de Santo Amaro. Arq. Hig. Saúde Pública, 8: 9-16, 1944.

7. COUTINHO, J. O. Contribuição ao estudo dos transmissores de malária no Distrito Federal, Brasil. Arq. Hyg., 12: 7-14, 1942.

8. COUTINHO, J. O. Contribuição para o estudo do An. (N.) tarsimaculatus no Distrito Federal, Brasil. Arq. Hyg., 13: 65 78, 1943.

9. COUTINHO, J. O. Anofelinos do Rio de Janeiro (Distrito Federal) com referência aos transmissores de malária. $O$ Hospital, 30: 651-62, 1946.

10. COUTINHO, J. O. Contribuição para o estudo da distribuição geográfica dos anofelinos do Brasil. São Paulo, 1947. [Tese — Faculdade de Medicina da USP].

11. COUTINHO, J. O. \& RICCIARDI, I. Transmissão da malária pelo An. tarsimaculatus Goeldi, 1905 em Bocaina no Litoral de São Paulo, Brasil. Arq. Hig. R. Janeiro, 13: 2732, 1943.

12. COVA-GARCIA, P.; PULIDO, J.; AMARISTA, R. J. Anopheles (Nyssorhynchus) emilianus Komp, 1941 en Venezuela. Rev. Venez. Sanid. Asist. Soc., 29: 27-32, 1964.

13. CURRY, D. P. Some observations on the Nyssorhynchus group of the Anopheles (Culicidae) of Panama. Am. J. Hyg., 15: 566-72, 1932.

14. DEANE, L. M. Malaria vectors in Brazil. Mem. Inst. Oswaldo Cruz, 81 (supl. II): 5-14, 1986. valo, no Estado do Rio de Janeiro. Tais hábitos certamente influenciam de forma negativa em sua capacidade vetorial na região Sudeste do País. Seu melhor desempenho como transmissor da malária é justificado, principalmente, por sua elevada densidade em certas épocas do ano (Deane ${ }^{14}, 1986$; Rachou $^{29}$, 1958).

15. DEANE, L. M.; CAUSEY, O. R.; DEANE, M. P. Notas sobre a distribuição e a biologia dos anofelinos das Regiões Nordestina e Amazônica do Brasil. Rev. Serv. Esp. Saúde Pública, 1: 827-963, 1948.

16. DEANE, L. M.; VERNIN, S. C.; DAMASCENO, R. G. Avaliação das preferências alimentares das fêmeas de Anopheles darlingi e Anopheles aquasalis em Belém, Pará, por provas de precipitina. Rev. Serv. Esp. Saúde Pública, 2: 793-808, 1949.

17. DYE, C. Vectorial capacity: must we measure all its components? Parasitol. Today, 2: 203-9, 1986.

18 FLORES-MENDOZA, C. \& DEANE, L. M. Where are the resting-places of Anopheles aquasalis males? Mem. Inst. Oswaldo Cruz, 88: 501-2, 1993.

19. FLORES-MENDOZA, C. Um estudo sobre alguns aspectos comportamentais, morfológicos e bioquímicos de Anopheles aquasalis Curry, 1932. Rio de Janeiro, 1994. [Dissertação de Mestrado - Instituto Oswaldo Cruz]

20. FONSECA, J. A. B. \& FONSECA, F. Transmissão da malária humana por anofelinos da Série Tarsimaculatus. Mem. Inst. Butantan, 16: 93-124, 1942.

21. FORATTINI, O. P. Entomologia médica. São Paulo, Ed. Univ. São Paulo, 1962. v. 1.

22. FREITAS, G. Pesquisas sobre a transmissão da malária na Baixada Fluminense. Rev. Med. Cirur. do Brasil, 50: $15-20,1942$

23. GABALDON, A. \& GUERRERO, L. An attempt to malaria by the weekly administration of pyrimethamine in areas of out-of-doors transmission in Venezuela. Am. $J$. Trop. Med. Hyg., 8: 433-9, 1959.

24. GALVÃO, A. L. A.; DAMASCENO, R. G.; MARQUEZ, A. P. Algumas observações sobre a biologia dos anofelinos de importância epidemiológica de Belém, Pará. Arq. Hig. R. Janeiro, 12: 51-111, 1942.

25. GARRET-JONES, C. Prognosis for the interruption of malaria transmission through assessment of the mosquito's vectorial capacity. Nature, 204: 1173-5, 1964.

26. GIGLIOLI, G. Malaria in British Guiana. Report. Dir. Med. Serv. (1938), Appendix II: 118-39, 1940.

27. LOURENÇO-DE-OLIVEIRA, R. \& HEYDEN, R. Alguns aspectos da ecologia dos mosquitos (Diptera: Culicidae) de uma área de planície (Granjas Calábria), em Jacarepaguá, Rio de Janeiro. IV. Preferências alimentares quanto ao hospedeiro e freqüência domiciliar. Mem. Inst. Oswaldo Cruz, 81: 15-27, 1986. 
28. NATAL, D. \& MARUCCI, D. Aparelho de sucção tipo aspirador para captura de mosquitos. Rev. Saúde Pública, 18 418-20, 1984.

29. RACHOU, G. R. Anofelinos do Brasil: comportamento das espécies vetoras de malária. Rev. Bras. Malariol. Doenç. Trop., 10: 145-81, 1958.

30. RACHOU, G. R.; MOURA-LIMA, M.; BARBOSA, A. L. Considerações sobre o An. (N.) tarsimaculatus Goeldi, 1905 (An. (N.) aquasalis, Curry, 1932) no Estado do Ceará, com especial referência ao seu encontro a $52 \mathrm{~km}$ da orla marítima. Rev. Bras. Malariol., 2: 57-65, 1950.

31. RAMOS, A. S. Observações sobre os anofelinos do litoral paulista Anopheles (Nyssorhynchus) tarsimaculatus (Goeldi, 1905), Anopheles (Nyssorhynchus) oswaldoi (Peryassu, 1922). Arq. Hig. Saúde Pública, 8: 51-2, 1943.
32. ROZEBOOM, E. L. Subspecific variations among neotropical anopheles mosquitoes, and their importance in the transmission of malaria. Am. J. Trop. Med., 22: 235-55, 1942.

33. SENIOR-WHITE, R. A. Studies on the bionomics of An. aquasalis Curry, 1932. Part III. Ind. J. Malariol., 6: 31-72, 1952.

34. SHANNON, R. C. Malaria studies in Greece: The reaction of Anopheline mosquitoes to certain microclimatic factors. Am. J. Trop. Med., 15: 67-81, 1935.

35. SIQUEIRA, A. F. Estudos sobre a reação de precipitina aplicada à identificação de sangue ingerido por triatomíneos. Rev. Inst. Med. Trop., 2: 41-63, 1960. 